\title{
El neoliberalismo educacional chileno y sus posibilidades de derrumbe en una nueva constitución: ¿Es posible?
}

\author{
Cristóbal Madero \\ Universidad Alberto Hurtado
}

\section{Resumen}

Chile enfrenta la tarea de redactar una nueva constitución que reemplace en plenitud la Constitución de 1980 creada durante la dictadura de Pinochet (1973-1990). En tal constitución se emplazan los pilares de un modelo neoliberal a partir del cual el sistema educativo escolar ha sido moldeado. En este artículo se exponen elementos acerca de cómo se construye la Constitución de 1980, su contexto histórico, y sus principales influencias. Se exponen además los principales elementos constitucionales relativos a la educación escolar, y cómo dichos principios se han traducido en una institucionalidad construida (y deconstruida en algún caso) entre los años 1980 y 2021. El artículo finaliza con una reflexión sobre la posibilidad del derrumbe de la arquitectura neoliberal del sistema educativo escolar chileno a propósito de la redacción de una nueva constitución en el país.

Palabras clave: Chile, sistema educativo, neoliberalismo, mercado, constitución politica 


\title{
Chilean Educational Liberalism and the Possibility to Overthrow it in the New Constitution: Is it Possible?
}

\begin{abstract}
Chile faces writing a new constitution that will fully replace the 1980 Constitution created during the Pinochet dictatorship (1973-1990). The 1980 Constitution was built upon a neoliberal model from which the school education system has been shaped. This article presents elements about how the 1980 Constitution was constructed, its historical context, and its main influences. The article also exposes the main constitutional features that impacted the educational system and how those features translated into institutions between 1980 and 2021. The article ends with a reflection on the possibility of the collapse of the neoliberal architecture of the Chilean school education system regarding the drafting of a new constitution in the country.
\end{abstract}

Keywords: Chile, educational system, neoliberalism, market, political constitution

\section{Le Néolibéralisme éducatif au Chili et ses possibilités d'écroulement et d'une nouvelle constitution, Est-ce possible?}

\section{Résumé}

Le Chili se trouve actuellement devant la possibilité d'écrire une nouvelle constitution qui remplacerait complètement celle de 1980 créée durant la dictature de Pinochet (1973-1990). La Constitution de 1980 était érigée selon un modèle néolibéral à partir duquel le système scolaire éducatif fut modelé. Cet article présente les éléments sur lesquels la Constitution de 1980 a été érigée, sur son contexte historique, et sur les principales influences auxquelles il a été exposé. L'article révèle également les principales caractéristiques constitutionnelles qui ont influencé le système éducatif et dont les traits caractéristiques ont été traduits en institutions entre 1980 et 2021. L'article se termine par une réflexion sur la possibilité de l'effondrement de l'architecture néolibérale du système éducatif scolaire chilien ainsi que la possibilité de la rédaction d'une nouvelle constitution pour le pays.

Mots-clés : Chili, système éducatif, néolibéralisme, marché, constitution politique

\section{Introducción}

El 25 de octubre del año 2020 Chile votó mayoritariamente aprobar la redacción de una nueva constitución para regir políticamente el Estado. Para ello, el 15 y 16 de 
mayo de 2021 eligió a 155 ciudadanos y ciudadanas para conformar la convención constitucional que llevará a cabo dicha tarea. Una constitución o carta fundamental cumple no solo un rol enunciativo sino uno de carácter performativo: la constitución no solo dice cosas, sino que las hace. La del año 1833, fue si bien no la primera cronológicamente, sí la primera que logró dar término a más de dos décadas de ensayos constitucionales desde la Independencia de Chile en 1810. La de 1925, hizo que el Estado se separase de la Iglesia. La de 1980, redactada durante la dictadura cívico-militar (1973-1990), hizo que Chile abrazase un modelo neoliberal de desarrollo. Es este modelo en el cual buena parte de los llamados derechos sociales se sostienen hoy.

El neoliberalismo se dice muchas maneras. Se trata de un concepto polisémico, orientador para algunos, e irritante para otros. Al hacer evaluación de los años dictatoriales (1973-1989) y post dictatoriales (1990-2021) en Chile, no pocos observadores señalan que lo que en dictadura se diseña e implementa, en democracia se institucionaliza (Bellei and Vanni 2015; Ruiz 2018). La profundidad de los cambios en el modelo de sociedad, en el modelo económico, y como consecuencia en la cultura chilena desde 1973 en adelante, produjo cambios estructurales que, si bien buena parte de la social democracia de los años 1990 y 2000 en Chile quiso transformar, no reunieron las voluntades suficientes para hacer los cambios radicales necesarios (Falabella 2021). Lo que ocurrió en el territorio de la seguridad social y de la salud, también se experimentó en el terreno de la educación.

Este artículo busca abordar preguntas: (i) ¿En qué consisten los principios constitucionales neoliberales de la Constitución de 1980?, (ii) ¿Cómo se han traducido dichos principios en instituciones y dispositivos de la educación escolar?, y finalmente (iii) ¿Podrá una nueva constitución demoler una ideología incrustada culturalmente en la sociedad chilena?

El artículo se organiza en cuatro partes. En una primera parte se da cuenta de cómo se construye la constitución de 1980, su contexto histórico, y sus principales influencias. En una segunda parte se exponen los principales elementos constitucionales relativos a la educación escolar. En una tercera parte se aborda de qué forma dichos principios se han traducido en una institucionalidad construida en lo que llamamos tres momentos: el dictatorial, el de transición, y el democrático. En una cuarta y final se plantea una reflexión con respecto a la posibilidad del derrumbe de la arquitectura neoliberal del sistema educativo escolar chileno a propósito de la redacción de una nueva constitución en el país.

\section{La constitución de 1980: Notas de contexto}

El 11 de septiembre de 1973, con la ayuda de las agencias de inteligencia de los Estados Unidos, el ejército chileno llevó a cabo un golpe de Estado contra el gobierno de Salvador Allende quien cumplía 1000 días en el cargo. El pueblo chileno eligió democráticamente a Allende como el primer presidente socialista con un programa 
llamado Camino Chileno al Socialismo. Allende continuó con la reforma agraria centralizada que los democristianos estaban promoviendo durante la presidencia de Eduardo Frei Montalva (1964-1970). Su plan de gobierno también incluyó la creación de un sistema de educación pública centralizado: la Escuela Nacional Unificada (ENU). En el contexto de la Guerra Fría, Estados Unidos interpretó su elección como una amenaza y se puso como objetivo el derribar al régimen a través de boicots económicos. La derecha política en Chile apoyó estos boicots y no interfirió con los planes estadounidenses. Fruto de tales acciones hizo que la inflación que se disparase dejando a la economía en una situación deplorable. Tales fenómenos sirvieron como base a la derecha para presionar a los militares a que tomaran el poder (Solimano 2012).

Como lo han documentado Valdés (Valdés 1995), O'Brien y Roddick (O’Brien and Roddick 1983), y Ebenstein (Ebenstein 2007), en las décadas previas al golpe, la Universidad de Chicago y la Pontificia Universidad Católica de Chile (PUC) firmaron un acuerdo en 1955 para mejorar la calidad de esa universidad. La primera clase llegó a Chicago en septiembre de 1956 (Soto 2012). Los estudiantes adquirieron el apodo de los Chicago Boys. Antes de encabezar la transformación de la economía a través de un proceso de terapia de choque que impactó en la salud, las pensiones y los sistemas educativos en las décadas de 1970 y 1980, el grupo reformó por primera vez la Escuela de Economía de la PUC a partir de 1958 (Centro de Estudios Públicos 1992). Durante la década de 1980, la escuela se convirtió en una extensión de las ideas neoliberales de la Escuela de Chicago.

Para el momento en que Jaime Guzmán, abogado, profesor de derecho en la PUC, e ideólogo de la Constitución de 1980, redactaba la nueva carta fundamental, Chile contaba con una base de políticas neoliberales llevadas a delante por un grupo de economistas que, posterior a la visita de Friedman en 1975, toman distintas posiciones en el gobierno. Principalmente entre 1975 y 1982 (Montecinos 1998), los Chicago boys asumen los Ministerios de Hacienda, Economía, Minas, Trabajo y Seguridad Social. Cuando no prestaban servicio en cargos públicos, ocupaban cargos académicos, principalmente en la PUC, o cargos corporativos en el sector privado. Friedman comenta en sus memorias que "gracias a su formación, nuestros estudiantes (los Chicago Boys) fueron casi los únicos economistas en Chile que no habían estado involucrados ni favorables al gobierno de Allende" (Friedman and Friedman 1998:398). Con respecto a la educación, ambos favorecieron la privatización del sistema educativo utilizando la retórica de modernización, desburocratización (Bafalluy 1983) o descentralización (Espinoza and González 1993).

La nueva Constitución hizo casi imposible cambiar una nueva estructura económica neoliberal, en la que los exalumnos chilenos de la Escuela de Economía de la Universidad de Chicago desempeñaron un papel importante. 


\section{La constitución y la educación}

Contar con una matriz neoliberal en la Constitución de 1980 no es sinónimo ni de que la provisión educativa escolar fuese a partir de ese año totalmente privada, ni tampoco que antes de 1980 no existieran opciones de educación privada. A mediados del siglo $\mathrm{XIX}$, mientras la educación era constitucionalmente una atención preferente del Estado, este y los privados se dividían en partes iguales la matrícula escolar (Labarca 1939). Más aún, hacia comienzos del siglo XX la educación privada va perdiendo protagonismo en la tasa de alumnos matriculados en comparación con la educación estatal. Se contabilizan 1207 escuelas fiscales a nivel primario y 547 particulares, de las cuales 187 pertenecían a la Iglesia católica (Brunner et al. 2006). Los años cincuenta marcan un hito en la historia de la educación en Chile. Por una parte, se aprueba una ley de subvenciones en tres etapas entre el año 1951 y 1958, la cual permitió que la educación privada se fuera extendiendo en números crecientes hasta nuestros días. Primero se subvencionó a los alumnos en un $50 \%$ de los costos que representaba su educación; luego se subvencionaron los sueldos de los profesores, y finalmente se apoyó con dineros fiscales los estudios en la Universidad Católica (privada, por cierto). Por otra parte, hacia mediados del siglo XX, la educación particular deja de depender del examen de la Universidad estatal, y pasa a depender de la Superintendencia de Educación. Según el Informe final del Primer Censo de la Educación Particular en Chile, en 1968, el 24\% de la educación primaria y secundaria correspondía a establecimientos privados, de los cuales el $52 \%$ eran de afiliación católica (CIDE 1971).

Para el año 1970, la educación deja de ser una atención preferente y pasa a ser una función primordial del Estado, según lo constata la reforma constitucional de ese año. El Estado tiene el deber de asegurar el derecho de los padres a educar a sus hijos en el centro educativo que consideren adecuado, y por ello el Estado debe asegurar la existencia de ofertas educativas diversas. La educación particular entra de lleno al sistema nacional de educación, aceptando cumplir con los planes y programas de la autoridad central, cuestión que en la práctica venía ya haciendo (CIDE 1971). Ahora bien, el debate que produjo esta consagración de la educación privada fue de proporciones, una vez que se le adhirió el adjetivo de pagada. Si bien por cuestiones de tipo ideológicas muchos criticaban la existencia de ofertas religiosas o seculares paralelas a la oferta estatal durante los siglos XIX y XX, en la práctica este tipo de educación era un aporte pues apoyaba una función pública y la gran mayoría de manera gratuita.

La ENU diseñada durante el gobierno de la Unidad Popular liderado por Allende, fue una de las importantes razones que llevó al quiebre democrático. Los debates sobre educación, y todo debate para el caso, quedaron interrumpidos por las casi dos décadas en que el dictador Pinochet estuvo en el poder.

La constitución de 1980 se caracterizó por consagrar, a puerta cerrada, el derecho a la educación y la libertad de enseñanza. Fuera de eso, los años que siguieron a la constitución fueron años de cambios normativos e institucionales muy profundos 
(Picazo 2013). Favoreciendo un proceso de descentralización, se traspasa toda la educación pública a los gobiernos locales o municipios. Se terminan además y de manera prácticamente exclusiva los subsidios a la oferta, y fiel a principios neoliberales se inaugura un sistema de subsidios a la demanda basado en la entrega de vouchers a las familias que, amparadas en el derecho a la educación de sus hijos, elegirían el colegio que más los satisficiera. La educación privada gratuita recibe la misma subvención que los alumnos de un centro educativo bajo administración municipal, dando de este modo el origen a la provisión conocida como particular subvencionada. Los colegios pagados quedan a su libre arbitrio, manteniéndose sí la exigencia de ajustar sus planes y programas según la reforma educacional de 1965.

\section{La educación neoliberal y sus momentos}

La constitución de 1980 tiene una matriz neoliberal que como hemos señalado no se despliega solo en los años de la dictadura, sino también posteriormente. Como consecuencia de los principios de la libertad de enseñanza y el derecho a la educación garantizados en la Constitución, pero sobre todo a partir de su interpretación, el Estado de Chile crea una institucionalidad que opera sin muchos contrapesos hasta la llegada de la democracia en 1990. A partir de 1990 y hasta hoy, si bien se corrigen algunos elementos de la institucionalidad pasada, se crean con más o menos conciencia, nuevas estructuras que en algunas de sus dimensiones siguen enclavadas en una matriz neoliberal. Siguiendo a Lipman (2009) habría momentos distintos en la aplicación del neoliberalismo al sistema escolar. Destacamos tres de esos momentos: (i) el momento dictatorial (1980-1989), (ii) el momento de la transición (1990-2006), y (iii) el momento democrático (2007-2021).

\section{El momento dictatorial}

Derecho a la Educación. El derecho a la educación en la constitución no expresa su sentido en cuanto derecho humano (United Nations 1948), sino al derecho que los padres tienen de educar a sus hijos y por tanto de elegir el establecimiento educacional para ello. Esto no es para nada una excepcionalidad, y no expresa 10 neolibera/tras tal derecho. Con frecuencia se siembra confusión sobre el concepto de elección en el debate sobre políticas educativas. Una aproximación ingenua podría abrazar irreflexivamente el que la elección de escuela, a nivel global, tiene su origen en la mente del inspirador del neoliberalismo en Chile, Milton Friedman, olvidando que la elección de escuela ha sido una bandera levantada desde el Movimiento por los Derechos Civiles, los afroamericanos y los hispanos en Estados Unidos, o las Iglesias en el caso de Chile. Alternativamente, otros con igual ingenuidad podrían elogiar a las escuelas particulares subvencionadas como un paradigma de la reforma de la privatización escolar y asociarlas con ideas neoliberales, olvidando que estas escuelas 
de facto funcionaban en Chile desde el siglo XIX, sin mediar obviamente la Constitución de 1980. En este sentido todos pueden encontrar un hogar bajo el paraguas de la elección escolar, pues esta no sería más que un mecanismo que coordina la demanda por y la oferta de establecimientos educacionales al interior del sistema educativo

Si bien este principio en el sentido del derecho a la elección de los padres estaba ya presente una década antes en la reforma constitucional de 1970, a partir de la Constitución de 1980 se ha interpretado como el derecho que los padres tienen no solo para elegir el establecimiento educacional para sus hijos, sino también el derecho a pagarpor tal educación. El Estado se asegura de que los padres paguen, o bien reciban un voucher para pagar. Este voucher que opera como subsidio a la demanda, tendría un uso exclusivo para pago en establecimientos educacionales. Si el apoderado no puede pagar, es el Estado quien le provee del recurso. Solucionada dicha asimetría de poder adquisitivo gracias al aporte del Estado, se solucionaría el problema. No habría nada de neoliberal en poder pagar por un servicio que en justicia es recibido.

Sin embargo, un problema profundamente neoliberal emerge en esta manera de traducir el derecho constitucional por la educación: no existe igualdad de condiciones entre los estudiantes pues no existen solo escuelas públicas con un costo fijo, sino también privadas de distinto coste que reciben el voucher, y privadas que no reciben el voucher. Se genera de esa forma un mercado de establecimientos educacionales compitiendo en el caso de los dos primeros, por atraer estudiantes. El voucher cumple el rol por una parte de estimular la competencia entre los oferentes de la educación. El voucher también puede funcionar como un incentivo al sostenedor para responsabilizarse de la permanencia de los estudiantes en los establecimientos educacionales. Más específicamente, opera como dispositivo para controlar la asistencia, uno de los elementos críticos al momento de prevenir, por ejemplo, la deserción escolar. Y si bien esto último es un incentivo bien localizado, lo que en la práctica ha sucedido, es un estímulo a la competencia.

Libertad de enseñanza. La constitución asegura también el derecho a la libertad de enseñanza. Si bien una interpretación de dicho derecho refiere a la libertad de cátedra universitaria en la educación superior, el uso más extensivo de dicho derecho está en la libertad para crear establecimientos educativos de manera de diversificar los tipos de proyectos educativos. Nuevamente, es del todo deseable, en una sociedad plural como la chilena, que existan distintas ofertas que aseguren alternativas para los padres: proyectos con énfasis en lo artístico, en lo religioso, en lo deportivo, entre otros. Sin embargo, el efecto de dicho derecho recae en que los establecimientos pueden elegir a los estudiantes que quieren educar.

La libertad de enseñanza trae de esta forma dos problemas. Por una parte, la idea de diversidad de proyectos solo está dirigida al mundo privado, y no al mundo de los establecimientos públicos a esa altura des municipalizados. Es decir, pareciera que la innovación y la creatividad están circunscritos al mundo de los emprendimientos en educación, es decir a la educación privada. Detrás de esto opera la mentalidad 
neoliberal, por la cual todo lo público sería por defecto algo poco atrayente, pues no tendría incentivos para hacer mejor su tarea. Curioso por decir lo menos en el mundo escolar dado que la misma constitución da espacio a la competencia vía vouchera las escuelas municipalizadas. Por otra parte, y más grave a nuestro parecer, mientras las escuelas públicas municipalizadas no podían seleccionar a sus estudiantes, las privadas con y sin subvención si lo podían hacer de acuerdo con criterios como la condición socioeconómica de los padres, las habilidades de los estudiantes, o la religión de la familia.

Con estos datos, si bien la matriz neoliberal orientaba al sistema educativo en una competencia individual por estudiantes, esta competencia estaba lejos de ser perfecta: padres con limitación de escuelas para elegir, y escuelas que elegían a sus estudiantes de acuerdo con criterios arbitrarios, y una camisa de fuerza a la educación pública para hacer más atractiva su oferta.

\section{El momento de la transición}

El tiempo de la transición entre la dictadura y la democracia, lo situamos entre 1990 y el año 2006. 2006 no es solo el año en que muere el dictador Pinochet (que luego de dejar el gobierno había continuado siendo Comandante en Jefe del Ejército, y luego de eso Senador), sino el año de la primera gran movilización de estudiantes secundarios por una mejor calidad de la educación. Se le bautizó públicamente como la movilización de los pingüinos (por los colores de uniforme escolar blanco con gris, o blanco con azul). Se trató de un año que marca los primeros 17 años de democracia, luego de 17 años de dictadura. Finalmente es el año en que comienza a gobernar por última vez de manera continua una presidenta (además mujer, la primera en la historia del país) del bloque de centro izquierda dando paso por primera desde la vuelta a la democracia, a la alternancia de bloques políticos en el poder. Por todo esto nos parece un buen rango de años donde situar el momento de la transición.

Si bien son muchas las políticas educativas que se desarrollan en los primeros tres gobiernos de la concertación ${ }^{1}$ (Patricio Aylwin 1990-1994, Eduardo Frei Ruiz-Tagle 1994-2000, y Ricardo Lagos 2000-2006), quisiéramos destacar la del financiamiento compartido de la educación escolar, y la de la prueba estandarizada SIMCE, que si bien es una prueba anterior (1988), cobra más y más protagonismo en los años de la transición.

\footnotetext{
1 Algunas de las reformas de estos años de transición son: (i) Se reforma el Estatuto Docente Ley 19.070 de 1991 el cuál para los docentes del sector municipal y para aquellos del sector particular subvencionado una jornada de 44 horas cronológicas, y una ratio de horas lectivas y no lectivas de 75/25; (ii) Se eleva la escolaridad obligatoria a 12 años en el año 2000; (iii) Se crea el marco para la buena enseñanza en el año 2003; (iv) Se implementa la evaluación docente
} 
Financiamiento Compartido. El financiamiento compartido es la política que en este periodo de transición democrática fue la más alineada a una concepción neoliberal de la educación, y no necesariamente por las razones más obvias ni previsibles desde el delicado balance político post dictadura en que se vivía. El financiamiento compartido buscaba, en un país lejos del desarrollo como era Chile en los primeros años de los 1990, insertar recursos al sistema educativo que el Estado por sí mismo no podía proveer. De esta forma el recurso a las familias se vio como la solución más apropiada. La idea del derecho a pagar por la educación ya estaba instalada en el sistema educativo como consecuencia del derecho a la educación examinado en la sección anterior. Sin embargo, el pago de los padres hecho en el marco del financiamiento compartido generaba una serie de consecuencias propias, desde todo punto de vista, de un modelo neoliberal.

En 1993 el financiamiento compartido se planteó como un mecanismo por el cual los establecimientos particulares no gratuitos pudieran cofinanciarse por apoderados y por el Estado en un esquema dinámico. Si el costo de la educación era de 100, familias podían pagar 10, y el Estado 90, o las familias 80, y el Estado 20. Si esto hubiera sido posible en cada establecimiento, hubiéramos asistido a uno de los mayores experimentos de integración social en Chile. Pero no fue este el caso pues no era cada establecimiento el que consideraba distintos montos privados y públicos de financiamiento, sino que cada establecimiento debía establecerse como uno que cobraba 20 o 30 o 50 de financiamiento compartido. De esa forma, se estableció un modelo de segregación casi perfecta. Colegios para pobres, para menos pobres, para clases medias pobres, y así. La decisión libre personal de cada apoderado, amparado por el derecho a la educación, lo podía llevar ahora a pagar colegios de más o menos financiamiento compartido.

El financiamiento compartido es un cambio estructural que, si bien hizo ingresar mayores recursos a la educación primaria y secundaria, no hay dudas de que fue un obstáculo más en el logro de una mayor integración social (Corvalán and GarcíaHuidobro 2016), tema que constituyó precisamente el centro del debate interrumpido en 1973. De hecho, esta medida generó aprehensiones dentro de la misma coalición gobernante que la impulsó, pues se temía que fomentara dinámicas de segregación y segmentación social del sistema escolar subvencionado por el Estado, lo cual atenta sin duda contra criterios de equidad.

Sistema de Medición de la Calidad de la Educación. Si bien el Sistema de Medición de la Calidad de la Educación (SIMCE) procede de una tradición de medición estandarizada de los aprendizajes en Chile, no fue hasta el final de la dictadura cuando esta se tornó en una pieza clave de la política educativa. Se trata de una política que si bien tradicionalmente ha encontrado rechazo desde mediados del siglo XX, este ha sido mayor una vez que desde los años de la transición comenzó a hacerse más relevante el resultado individual de cada estudiante (Falabella and de la Vega 2016). Se trató en un comienzo de pruebas censales de lectura y matemática para 4 tos básicos, 8 vos básicos, y 2 do medio, lo cual fue incrementándose en asignaturas y en niveles. 
De manera más dramática a partir del año 2006, las pruebas se incrementan significativamente.

Medir si los estudiantes aprenden o no es, desde el punto de vista del Estado, una responsabilidad basada en un principio de justicia: no es justo que unos niños aprendan más que otros. Ahora bien, el buen uso que se puede dar a la medición sirvió a otros propósitos anclados en el individualismo metodológico clásico (Becker 1964) en el diseño neoliberal de sociedad. Esa misma medición que puede servir el propósito de cerrar brechas, puede ser usada como herramienta de castigo o premio a una escuela o a un profesor. También puede estigmatizar escuelas, que, a su vez, siendo las más necesitadas de buenos docentes, los alejan.

\section{El momento democrático}

El momento democrático lo establecemos a partir del año 2007. Las movilizaciones de estudiantes secundarios en el año 2006, y las de universitarios el 2011, van remeciendo la estructura de la institucionalidad chilena. La sociedad se va haciendo más democrática, escuchando voces más allá de la representación ciertamente democrática ya dada por los partidos. Una sociedad civil reconstruida, movimientos y movilizaciones van dando cuerpo a una democracia más plena, que entre otras cosas es capaz entre los años 2019 y 2021 de haber catalizado el descontento social en la redacción de una nueva constitución y no en violencia incontrolable. Durante este momento el sistema educativo sigue creando instituciones con lógica neoliberal mercantil, al tiempo que es capaz, por el peso de la evidencia y de la historia de desarmar otras. Aquí destacamos la creación de dos instituciones, la Agencia de la Calidad de la Educación, y el Sistema de Desarrollo Profesional Docente como parte de la continuidad en la creación de nuevas instituciones con algunos tintes neoliberales. En la parte final, nos referiremos al desarme de parte de la institucionalidad, signo preclaro de la crisis que experimenta el neoliberalismo aplicado a la educación.

\section{La agencia de la calidad de la educación}

Uno de los reclamos de los estudiantes secundarios en las movilizaciones de 2006, fue la exigencia de una mayor calidad de la educación. No se trató de la principal de sus exigencias, pero sin duda fue una que fue tomando más y más protagonismo. El gobierno de Michelle Bachelet (2006-2011) convoca a un comité asesor para procesar los temas que emergieron de la movilización. Entre otras cosas eso deriva en una nueva Ley General de Educación en el año 2011. Junto con ello, se planteó la necesidad que el Estado chileno tenía de asegurar mayor calidad en el sistema educativo. Esto venía ya siendo planteado tanto por OCDE (grupo de países al que 
Chile se había integrado recientemente), y por el Banco Mundial. Asegurar calidad y equidad se volvían una condición de posibilidad de desarrollo.

La ley 20.529 crea el "Sistema Nacional de Aseguramiento de la Calidad de la Educación Parvularia, Básica y Media y su Fiscalización”, que comprende la creación de dos instituciones: la Superintendencia de Educación, y la Agencia de la Calidad de la educación. La primera tiene por función fiscalizar, mientras la segunda evaluar y orientar al sistema educativo. La Agencia de la Calidad es una institución que en su rol evaluador y orientador debe por ley generar un ordenamiento de los establecimientos escolares en Chile de acuerdo con su rendimiento tanto en la prueba SIMCE (un 67\% se establece de acuerdo a los niveles de aprendizaje en esta prueba), en indicadores de desarrollo personal y social (un 33\% en dimensiones como hábitos de vida saludable, clima de convivencia, puntaje SIMCE nuevamente, etc.) , y considerando factores correctores de la desigualdad inherente al sistema educativo (escolaridad de la madre, índice de vulnerabilidad, etc.) De esta forma cada escuela obtiene un indicador que la determina como un establecimiento de desempeño alto, medio, medio-bajo, o insuficiente.

Querer asegurar calidad y crear una institución para ello es necesario y justo. Pero puede ser también, si es creado bajo una lógica neoliberal, problemática. La agencia de la calidad tiene por mandato acompañar a los establecimientos que quedan categorizados como insuficientes, para evitar que se mantengan en la insuficiencia por más de cuatro años, pues eso la llevaría a su cierre o pérdida del reconocimiento oficial del ministerio de educación. La prueba SIMCE a la que nos referíamos en la sección anterior, es una prueba estandarizada, que fijaría en gran parte lo que es y lo que no es calidad. De acuerdo con dicha definición, se organizarían las prácticas y comportamientos al interior de la escuela (Ball 2008). La calidad que se busca asegurar es definida centralmente, lo cual si bien puede obedecer a un genuino interés de justicia (que todos aprendan lo mismo asegurando que nadie se quede atrás), reduce la educación a lo medible de manera estandarizada, y la coordinación del sistema educativo a principios gerenciales o de New Public Management (Falabella 2015; Maldonado and Chávez 2020).

Junto con esto, la Agencia de la Calidad busca proveer de más información a los padres y apoderados sobre los resultados obtenidos por sus hijos, de modo de hacer su elección de escuela más informada. Nuevamente, en el contexto del mapa de la institucionalidad que hemos venido revisando, fomentar el mercado educacional y la competencia dentro de él, hace de esta política de dotar de más información, una política que de no haber cambios a la política de vouchers, solo castigará a las escuelas más disminuidas en sus resultados, que por lo general son además las más pobres.

\section{El sistema de desarrollo profesional docente}


El techo de la calidad de un sistema educativo es la calidad de sus docentes. Para el año 2016, Chile crea la Ley de Desarrollo Profesional Docente, una promesa de mayor profesionalización en muchos ámbitos y un avance con respecto a los estadios anteriores del ejercicio de la docencia. Los más de 240.000 docentes que ejercen en las escuelas chilenas hoy, son susceptibles de enfrentar con esta ley una carrera, en el sentido de proyectar su desarrollo profesional, cosa hasta el momento ausente en la legislación chilena. En efecto el docente que ejerce en la educación pública, y paulatinamente los de la educación particular subvencionada, transitan a través de cuatro tramos obligatorios ${ }^{2}$, según sus años de experiencia pedagógica, los resultados del portafolio de la evaluación docente, y los resultados en evaluación de conocimientos disciplinarios y pedagógicos. La ley también mejora las condiciones del trabajo al aumentar la proporción de horas no lectivas desde un $25 \%$ como lo establecía el Estatuto Docente, a un $35 \%$ en todos los establecimientos, y hasta a un $40 \%$ en establecimientos con altos niveles de vulnerabilidad. Otra política asociada a esta mejora de condiciones es el proceso de inducción al cual entran los docentes durante su primer o segundo año de ejercicio profesional por 10 meses. Esto no solo facilita la inserción, sino que otorga cierto sentido de comunidad a la docencia. Un tercer elemento, aunque ya estaba asegurado previamente, es el derecho de cada docente a la formación en servicio provista por el CPEIP de forma directa o con ayuda de universidades y otras instituciones sin fines de lucro certificadas.

Existen al menos dos elementos de esta ley que son construidos desde una lógica neoliberal. El primero es que, si bien la mentoría generaría algún sentido comunitario, todos los incentivos a avanzar de tramo en tramo son individuales. No existen incentivos grupales en la carrera docente. Junto con ello, la remuneración se incrementa a partir de bonos que como lo indica el nombre, no son parte de un sueldo fijo, lo cual hace que las rentas sean variables. El incremento del bono de reconocimiento profesional y la asignación de tramo de la carrera, y el de reconocimiento por ejercer en establecimientos con alta vulnerabilidad, son incentivos individuales, y que hoy pueden estar y mañana no. Finalmente, la remuneración depende de la evaluación docente lo cual enfatizaría el control gerencialista de la docencia, haciéndola menos profesional (Ruffinelli 2016). La docencia es la única profesión evaluada de esta manera en Chile. Las otras profesiones son reguladas a partir de la acción colegiada del grupo profesional.

\section{¿El derrumbe de lo neoliberal en la educación chilena?}

La lógica neoliberal en educación ha estado presente en los últimos 40 años en Chile. Ha definido instituciones, reglas del juego, modos de entender la profesión docente, y en qué consiste liderar una escuela, por mencionar solo algunos elementos. Esta

\footnotetext{
${ }^{2}$ Inicial: 0 a 4 años - Avanzado: tras 5 años - Experto I: tras 10 años - Experto II: tras 15 años.
} 
lógica no ha dejado de ser cuestionada en estos 40 años, y no han sido pocos los espacios en que la lógica ha cedido a la evidencia y al peso de la historia.

Hoy, año 2021, gracias a la Ley de Inclusión de 2015, el financiamiento compartido se va acabando, haciendo que más del $90 \%$ de los establecimientos educativos sean gratuitos en Chile. Gracias a esa misma ley, más del $90 \%$ de los establecimientos ya no pueden seleccionar a sus estudiantes: son las familias, las que, por primera vez en la historia de Chile, pueden seleccionar el colegio, sin poderles ser negado el acceso gracias a un nuevo Sistema de Admisión Escolar. La Ley de Inclusión prohíbe además el lucro de los sostenedores de los establecimientos lo cual hace que, si bien el sistema sigue operando con un sistema de financiamiento a la demanda y regulado por la asistencia, son mucho menores los incentivos a aquellos, que sin ser ni de cerca una mayoría, hicieron de la educación un negocio con todas las reglas del juego a su favor por más de 30 años en el país.

Si bien las pruebas SIMCE siguen siendo relevantes, y es el principal dispositivo en torno al cual se organiza la labor evaluativa y orientadora de la Agencia de la Calidad de la Educación, estás han disminuido desde 2016 en el modo (censal o muestral), y en los niveles educativos en que se aplican, así como en el número total de pruebas al año. Son pasos hacia un sistema menos centrado en la estandarización, pero aún lejos de un cambio sustancial en esta materia.

Claramente lo neoliberal no se ha derrumbado al hablar del sistema educativo chileno. ¿Podrá una constitución transformar los principios desde una ideología neoliberal a una más comunitaria, y que eso redunde en modos distintos de entender la educación y la organización del sistema educativo? ¿Podrá una nueva constitución demoler una ideología incrustada culturalmente, en la subjetividad de cada chilena y chileno?

La constitución a nuestro entender puede claramente señalar caminos que permitan pensar una educación menos anclada en principios neoliberales. Y para eso la misma definición de derecho a la educación debe ser reformada en la constitución (Gonzalez et al. 2020). Como lo han señalado no pocos en el contexto preliminar de la discusión constitucional, el derecho a la educación no puede seguir siendo un derecho a pagar por un establecimiento que yo elijo (lo cual a pesar de la gratuidad del más de $90 \%$ de establecimientos se puede seguir haciendo en los establecimientos privados con pago). Sin duda que tiene que haber reforma de instituciones y normativas, pero es la misma definición aquello de lo que adolecemos como sociedad. Es un tiempo más de política que de políticas, en el sentido fuerte del término.

En tal sentido, el sistema educativo en Chile, orientado desde una nueva Constitución Política puede orientar con una clara definición de derecho a la educación, al sistema político y educativo en su conjunto. Tal definición debiera incluir elementos básicos como es la duración del proceso educativo (restringido a tiempo acotado, o a lo largo del a vida), así como las dimensiones que abordará dicho proceso (dimensiones cognitivas, valóricas, y de habilidades), y por supuesto los fines de dicha educación. Igualmente debiera indicar con claridad las responsabilidades de quienes deberán proteger, promover, y asegurar tal derecho. Sin duda es el Estado 
quien tiene dicha responsabilidad en primer lugar, pero lo pregunta será por el tipo de Estado. Hasta ahora lo ha sido uno de tipo de subsidiario. ¿Cambiará? El camino, lento pero sistemático, de derrumbe del neoliberalismo en educación en Chile, indica que la Convención Constitucional dada su composición, no debiera desviarse de tal camino de cambio y transformación.

Una vez desarrollada la o las definiciones del derecho a la educación, la nueva constitución podría orientar el sistema educativo hacia la tarea todavía titánica que significaría seguir derrumbando inteligentemente la estructura neoliberal de las instituciones y normativas del sistema educativo escolar chileno. No es una tarea nada fácil, pero tal vez como nunca había habido tantas voluntades y sentido de país, como para continuar tal tarea con decisión.

\section{Referencias}

Bafalluy, Alfredo Prieto. 1983. La Modernización educacional. Ediciones Universidad Católica de Chile.

Ball, S. J. 2008. "The Legacy of ERA, Privatization and the Policy Ratchet." Educational Management Administration \& Leadership 36(2):185-99. doi:

10.1177/1741143207087772.

Becker, Gary. 1964. Human Capital. A Theoretical and Empirical Analysis with Special Reference to Education. Chicago ; London: The University of Chicago Press.

Bellei, Cristián, and Xavier Vanni. 2015. "Evolución de Las Políticas Educacionales En Chile: 1980 - 2014." Pp. 23-45 in El Gran Experimento: Mercado y Privatización de la Educación Chilena. Santiago, Chile: LOM Ediciones.

Brunner, José Joaquín, Gregory Elacqua, Soledad Gonzalez, Ana M. Montoya, and Felipe Salazar. 2006. Calidad de la educación, claves para el debate: la organización de los sistemas escolares en el mundo contemporáneo. Santiago, Chile: RIL Editores.

Centro de Estudios Públicos. 1992. El Ladrillo: Bases de La Politica Economica Del Gobierno Militar Chileno. Santiago, Chile: CEP.

CIDE. 1971. La Educación Particular En Chile. Santiago, Chile: CIDE.

Corvalán, Javier, and Juan E. García-Huidobro. 2016. "Eucación y Mercado: El Caso Chileno." Pp. 17-56 in Mercado escolar y oportunidad educacional. Libertad, diversidad y desigualdad, edited by J. Corvalán, A. Carrasco, and J. E. GarcíaHuidobro. Santiago, Chile: Ediciones UC.

Ebenstein, Lanny. 2007. Milton Friedman. A Biography. New York, NY: Palgrave Macmillan.

Espinoza, Oscar, and Luis Eduardo González. 1993. "La Experiencia Del Proceso de Desconcentración y Descentralización Educacional En Chile 1974-1989." Santiago: PIIE.

Facultad de Educación UAH. 2020. Educación y Constitución: 10 ldeas Fundamentales. Santiago, Chile: Universidad Alberto Hurtado. 
Falabella, Alejandra. 2015. "El mercado escolar en Chile y el surgimiento de la nueva gestión pública: el tejido de la política entre la dictadura neoliberal y los gobiernos de la centroizquierda (1979 a 2009)." Educação \& Sociedade 36(132):699-722. doi: 10.1590/ES0101-73302015152420.

Falabella, Alejandra. 2021. "Going Left or Right? A Study of the Policy Rationale of the Chilean Center-Left Coalition Concertación in Education." Education Policy Analysis Archives 29(5):1-28. doi: 10.14507/epaa.29.5266.

Falabella, Alejandra, and Luis Felipe de la Vega. 2016. "Políticas de responsabilización por desempeño escolar: Un debate a partir de la literatura internacional y el caso chileno." Estudios pedagógicos (Valdivia) 42(2):395-413. doi: 10.4067/S071807052016000200023.

Friedman, Milton, and Rose D. Friedman. 1998. Two Lucky People: Milton and Rose D. Friedman Memoirs. Chicago, IL; London, UK: The University of Chicago Press. Gonzalez, Javier, Rafael Carrasco, Canela Bodenhofer, and Vicente Silva. 2020. Constituyamos Otra Educación, Una Mejor Sociedad Es Posible: 10 nudos constitucionales en educación y experiencias internacionales destacadas para inspirar el debate nacional. SUMMA y GI-ESCR.

Labarca, Amanda. 1939. Historia de La Enseñanza En Chile. Santiago, Chile: Editorial Universitaria.

Lipman, Pauline. 2009. "Paradoxes of Teaching in Neo-Liberal Times: Education 'Reform' in Chicago." Pp. 67-80 in Changing Teacher Professionalism: International Trends, Challenges and Ways Forward. New York, NY: Routledge.

Maldonado, Cristian Oyarzún, and Rodrigo Cornejo Chávez. 2020. "Trabajo docente y nueva gestión pública en chile: Una revisión de la evidencia." Educação \& Sociedade 41:e219509. doi: 10.1590/es.219509.

Montecinos, Verónica. 1998. Economists, Politics and the State: Chile 1958'1994. Amsterdam: CEDLA.

O'Brien, Phil, and Jackie Roddick. 1983. Chile: The Pinochet Decade. The Rise and Fall of the Chicago Boys. London: Latin American Bureau.

Picazo, María I. 2013. Las Políticas Escolares de La Concertación Durante La Transición Democrática. Santiago, Chile: Ediciones Universidad Diego Portales.

Ruffinelli Vargas, Andrea. 2016. "Ley de desarrollo profesional docente en Chile: de la precarización sistemática a los logros, avances y desafíos pendientes para la profesionalización." Estudios pedagógicos (Valdivia) 42(4):261-79. doi: 10.4067/S0718-07052016000500015.

Ruiz, Carlos. 2018. "Educación y Política En La Transición Chilena.” Pp. 139-61 in Privatización de lo público en el sistema escolar. Chile y la agenda global de educación, edited by C. Ruiz, L. Reyes, and F. Herrera. Santiago, Chile: LOM Ediciones.

Solimano, Andrés. 2012. Chile and the Neoliberal Trap. Cambridge, UK: Cambridge University Press.

Soto, Angel. 2012. "Entrevista a Sergio de Castro: El Profesor Friedman y La Vigencia de Sus Ideas." Pp. 73-84 in Un Legado de Libertad. Milton Friedman en Chile. 
Santiago, Chile: Instituto Democracia y Mercado/Atlas Economic Research Foundation/Fundacion para el progreso.

United Nations. 1948. "La Declaración Universal de Derechos Humanos | Naciones Unidas." United Nations. Retrieved May 26, 2021 (https://www.un.org/es/aboutus/universal-declaration-of-human-rights).

Valdés, Juan G. 1995. Pinochet's Economists: The Chicago School in Chile. Cambridge, UK: Cambridge Universtity Press. 